\title{
Distribution and national conservation status of the lichen family Lobariaceae (Peltigerales): from subtropical luxuriant forests to the alpine scrub of Nepal Himalaya
}

\author{
Devkota $S^{1 *}$, Keller $C^{1}$, Olley $\mathbf{L}^{2}$, Werth $S^{1,3}$, Chaudhary $\mathbf{R P}^{4}$ and Scheidegger $\mathrm{C}^{1}$ \\ ${ }^{1}$ Swiss Federal Research Institute WSL, CH-8903 Birmensdorf, Switzerland \\ ${ }^{2}$ Royal Botanic Gardens, Edinburgh (RBGE) EH3 5LR, Scotland, UK \\ ${ }^{3}$ Institute of Plant Sciences, University of Graz, 8010 Graz, Austria \\ ${ }^{4}$ Research Centre for Applied Science and Technology (RECAST), Tribhuvan University, Kirtipur, Nepal
}

Devkota S, Keller C, Olley L, Werth S, Chaudhary RP, Scheidegger C 2017 - Distribution and national conservation status of the lichen family Lobariaceae (Peltigerales): from subtropical luxuriant forests to the alpine scrub of Nepal Himalaya. Mycosphere 8(4), 630-648, Doi $10.5943 /$ mycosphere/8/4/10

\begin{abstract}
During 2007 - 2014, voucher specimens of Lobariaceae were collected from different geographic locations of Taplejung, Solukhumbu, Rasuwa, Gorkha, Manang, Kaski, and Myagdi districts of Nepal. Morphological characters, chemical tests and thin-layer chromatography techniques (TLC) were applied for the identification. Combining with earlier publications on Lobariaceae, this study summarized two genera Lobaria and Sticta each with seven and six species, reported from ten different districts of Nepal. The altitudinal distribution of the species varies from $1350 \mathrm{~m}$ to $5004 \mathrm{~m}$ (i.e. subtropical to alpine bioclimatic zones) above sea level, from Eastern, Central and Western parts of Nepal. Lobaria adscripturiens (Nyl.) Hue, L. fuscotomentosa Yoshim. L. aff. quercizans Michx. and S. limbata (Sm.) Ach. are new records for the lichen flora of Nepal. Major chemotypes and their distributions are presented and further work on molecular analyses of these specimens from Nepal is recommended for the understanding of their systematic position. Extensive exploration covering more geographical areas of Nepal will increase the understanding on taxonomy and ecology of this interesting lichen group. In the light of species occurrence and following IUCN criteria, we have also prepared the national conservation status of Lobariaceae species, which could provide further insights for the inclusion of species in national conservation priorities.
\end{abstract}

Key words - Checklist - Lobaria - Sticta - Taxonomy - TLC

\section{Introduction}

A lichen is a self-supporting, symbiotic organism where fungal (mycobiont) and algal or cyanobacterial cells (photobiont) partners exist and function together (Honegger 1991, Kirk et al. 2008). Recently, basidiomycetous yeasts were reported as additional components of lichens (Spribille et al. 2016), or as lichenicolous fungi (Millanes et al. 2016). Lichens are cosmopolitan and equally able to colonize a variety of terrestrial habitats including those which are inhospitable for other organisms such as rock surfaces, the coldest alpine peaks (Wirtz et al. 2003) and deserts. The primary 
photobiont, (green-algal or cyanobacterial) fixes carbon for both partners (Søchting 2015). The total number of lichenized fungi is still unknown, but different researchers predicted or estimated the number between 13,500 - 28,000 species (Hawksworth et al. 1995, Kirk et al. 2008, Lücking et al. 2009). The Himalayan belt with a variety of terrestrial habitats is considered as a lichen rich landscape of Asia, comprising different forms of lichens (Upreti 1998). Lichen exploration and identification in Nepal is still poorly developed compared to higher plants, so more exploration and investigations are required to learn more about lichenized fungi (Olley \& Sharma 2013, Søchting 2015). Proper understanding of the distribution and abundance of lichens will help in the preparation of a national Red list, crucial for the successful planning of nature conservation (Scheidegger \& Goward 2002).

The Lobariaceae, second largest family of Ascolichens belongs to the order Peltigerales in the class Lecanoromycetes with about 14 genera and about 400 species (Kirk et al. 2008, Lumbsch \& Huhndorf 2010, Moncada et al. 2013, 2014, de Lange \& Galloway 2015). The scientific identification and publications on Lobariaceae from Nepal goes back to the work of the Japanese scientist Yasuhiko Asahina from National Science Museum, Tokyo, when he identified specimens collected by Japanese expedition members in Nepal Himalaya during 1952 - 1953 (Asahina 1955). Only a few publications (Asahina 1955, Awasthi 1960, 2007, Yoshimura 1971, Sharma 1979a, b, 1995, Joshi \& Awasthi 1982, Baniya et al. 2010, Olley \& Sharma 2013, Devkota et al. 2014) have mentioned the occurrence of Lobariaceae in Nepal.

Several ethnic uses of Lobariaceae were reported by numerous researchers around the globe, though there is not a single publication on uses of these taxa in Nepal. Some of the important uses of Lobaria spp. are the medical treatment of: skin diseases - Bhutan (Søchting 1999); indigestion Yunnan (Ju et al. 2013); arthritis - Canada (Johnson 1997); sores on the leg - USA (Densmore 1939); L. pulmonaria in lung ailments during the early modern era - Europe (Cameron 1900, Llano 1948); cuts - Italy (Guarrera et al. 2008); coughing up blood - Canada (Turner \& Efrat 1982); L. isidiosa to treat inflammation and burns - China (Hu et al. 1980); L. orientalis in lung ailments - China (Wang \& Qian 2013); L. quercizans for the tonic effect - USA (Smith 1923); L. retigera and L. yunanensis for indigestion, burns - China (Wang \& Qian 2013); S. nylanderiana for excessive cough - India (Sinha \& Singh 2005) and S. wrightii for indigestion - China (Wang \& Qian 2013). Recently, Devkota et al. (in prep. 2017) studied trade value of lichens in Nepal Himalaya and found L. retigera and $S$. nylanderiana for their cross-border trade value.

The main objective of this research was to explore and record the geographic distribution of Lobariaceae from different bioclimatic zones of Nepal. In addition, drawing on previously published data, we prepared an updated checklist of Lobariaceae with national conservation status for Nepal.

\section{Materials \& Methods}

This study is based on the specimens collected during three major lichenological field expeditions in 2007, 2009 and 2011 - 2014 in seven different districts from the subtropical zone (1000 $\mathrm{m}-2000 \mathrm{~m}$ ) to the nival zone (5000 $\mathrm{m}$ and above) of Nepal (Fig. 1).

Louise Olley (RBGE) and her team conducted an expedition to Langtang National Park, Rasuwa district in 2007 and collected c. 1200 macro and microlichens. Afterwards, in 2009 Christoph Scheidegger and Shiva Devkota visited Langtang National Park and collected c. 508 Lobariaceae specimens. The major expeditions in Gorkha district, and Sagarmatha and Kangchenjunga areas conducted during 2011 - 2013 years by Christoph Scheidegger and his team, resulted in the collection of c. 4200 specimens of lichens in the framework of a Nepal - Swiss Collaborative Research Project. Research transects were laid out to document vascular plants, macromycetes, lichens, birds and butterflies (Scheidegger et al. 2010, Katuwal et al. 2016, Rai et al. 2016). 


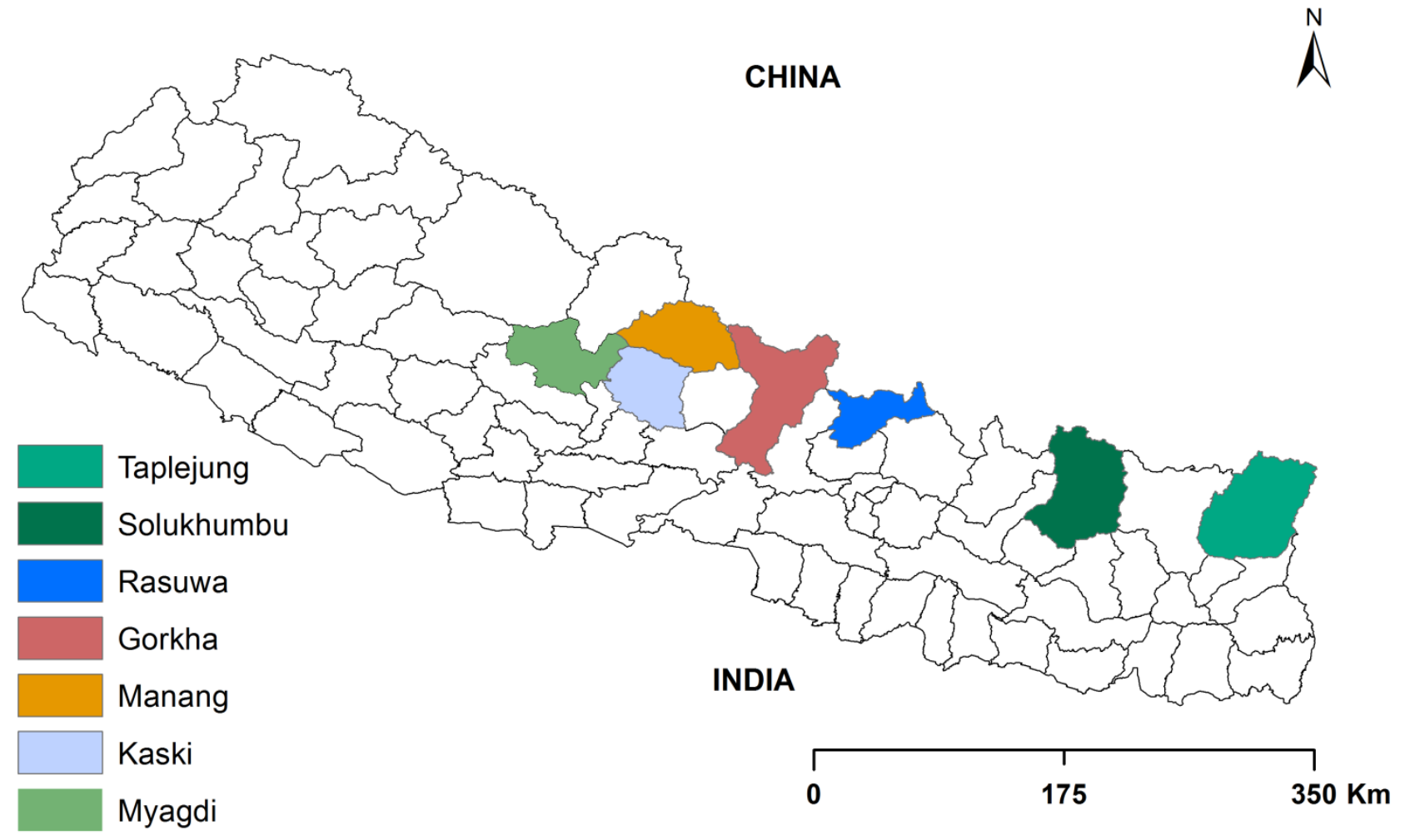

Fig. 1 - Map of Nepal showing seven study districts.

The following areas were visited for intensive specimen collecting: Olangchung Gola and Ghunsa valleys of Kangchenjunga Conservation Area, Taplejung district; Dudhkunda and Dudhkoshi valleys of Sagarmatha National Park, Solukhumbu district; Gosaikunda and Kyanjin valleys of Langtang National Park, Rasuwa district; and Nubri and Tsum valleys of Manaslu Conservation Area, Gorkha district. Manang, Myagdi and Kaski districts were visited to record the occurrence of taxa along walking trails only (Fig. 1). Kaski, Manang and Myagdi districts were visited once in 2014, Rasuwa was visited twice in 2007 and 2009. The other valleys were repeatedly visited once in a year between 2011and 2013. Collected and dried specimens were identified by studying their morphology, anatomy, and using color spot tests with $\mathrm{K}, \mathrm{C}$, and $\mathrm{P}$ at the lab of Central Department of Botany, Tribhuvan University, and Kathmandu, Nepal and at the Royal Botanic Gardens, Edinburgh, Scotland.

Unidentified specimens were then brought to lab at the Swiss Federal Research Institute, WSL, Zurich, Switzerland for the identification of chemical substances with thin-layer chromatography (TLC) (Culberson \& Kristinsson 1970, Culberson 1972). Acetone was used to extract secondary substances from $0.2 \mathrm{~g}$ of cleaned thallus portions for 10 minute at $40^{\circ} \mathrm{C}$. Extractions were then spotted on Merk silica gel F254 plates. Solvent C system i.e. Toluene: Acetic acid (200:30 $\mathrm{ml}$ ) was used to elute substances for about 30-35 minutes in a closed glass cabinet. Plates were air dried for 30 minutes and then observed under short wavelength $(\sim 254 \mathrm{~nm})$ UV light. Finally, plates were immersed carefully in a sulphuric acid bath, air dried for two to five minutes and heated in an oven at $100^{\circ} \mathrm{C}$ for 15 minutes. Known references were used as control substances to identify chemical substances.

A set of identified specimens from 2007 explorations are housed in the Department of Plant Resources (DPR), Ministry of Forests and Soil Conservation, Nepal and specimens from 2009 - 2014 explorations are deposited at Tribhuvan University Central Herbarium (TUCH), Kirtipur, Kathmandu, Nepal. Nomenclature of the species follows the MycoBank Database (2017; http://www.mycobank.org/). Conservation status of lichens for the local scale is determined following the combination of recommended guidelines for the application of IUCN criteria 
(Scheidegger et al. 2000, Scheidegger \& Goward 2002, Dahlberg \& Mueller 2011), interpretation methods of IUCN Red List categories for cryptogams (Hodgetts 2000) and, expert opinion (Nascimbene et al. 2013). Two different terms, local and regional, were assigned to the spatial scales (Cornell \& Lawton 1992). Each species was given a threat category: critically endangered (CR), endangered (EN), vulnerable (VU), least concern (LC) and data-deficient (DE) on the basis of criterion D (rarity) and criterion B (geographic range) (IUCN Standards and Petitions Subcommittee 2010, Nascimbene et al. 2013).

Critically endangered (CR): For a species known from a single locality of Nepal.

Endangered (EN): For a species known only from one to two regions of Nepal in more than one locality.

Vulnerable (VU): For a species known from three to five regions of Nepal in more than one locality. Least concern (LC): For a species known from more than five regions of Nepal if it neither suffered serious decline nor had a restricted area of occurrence.

Data deficient (DC): For a species with insufficient data, where additional data are needed to determine the IUCN Red List status.

Secondary data were collected by consulting prior research papers on lichens of Nepal, wherever Lobariaceae species were mentioned. Their geo-reference and distribution range is noted (if available). Symbols E, C, W represent East, Central and West parts of Nepal respectively

\section{Results \& Discussion}

In total, 2386 specimens were identified from seven districts of Nepal. Prior to this work, nine species of Lobariaceae (four species of Lobaria; five species of Sticta) were recorded from Nepal and with this work, the family Lobariaceae represents 13 species (seven species of Lobaria; six species of Sticta) in Nepal. During the Rasuwa visit (2007), Louise Olley and her team had listed $L$. adscripturiens (Nyl.) Hue, L. fuscotomentosa Yoshim. and S. limbata (Sm.). Similarly, Christoph Scheidegger and his team have also collected the same species including L. aff. quercizans Michx during their expeditions in 2009 - 2014 to different parts of Nepal. These species had not been published with ecological, chemical and morphological information till then. After comprehensive morphological and chemical studies, we confirmed the above four species as new additions to the lichen flora of Nepal.

Apart from the districts we visited, Panchthar, Dolakha, and Doti districts were also reported to harbor species of Lobaria and Sticta. Yoshimura (1971) reported L. pseudopulmonaria (= L. isidiosa) from Panchthar and Kurokawa (1967) reported L. subretigera from Rolwaling Himalaya, Dolakha, but according to Yoshimura (1971) this species is synonymous with L. pseudopulmonaria. Sharma (1979a) reported S. platyphylloides, S. praetextata, L. discolor and L. retigera from Khaptad, Doti. In summary, Lobariaceae species were recorded from ten districts mostly from the eastern part of Nepal (Fig. 2).

The TLC technique allowed to distinguish the two species pairs L. pseudopulmonaria / L. isidiosa and Lobaria kurokawae / L. retigera showing that the presence of stictic and norstictic acids are not taxonomically important but that the presence of the retigeric and retigeranic acids can discriminate between the two taxa. Both taxa include multiple ITS haplotypes which include isidiate and apotheciate specimens (Cornejo et al. 2009). A more detailed phylogenetic analysis of the cyanobacterial taxa belonging to Lobaria is needed for an appropriate taxonomic circumscription of these taxa. In this paper, we recognize the two chemically distinct taxa $L$. isidiosa and L. retigera. 


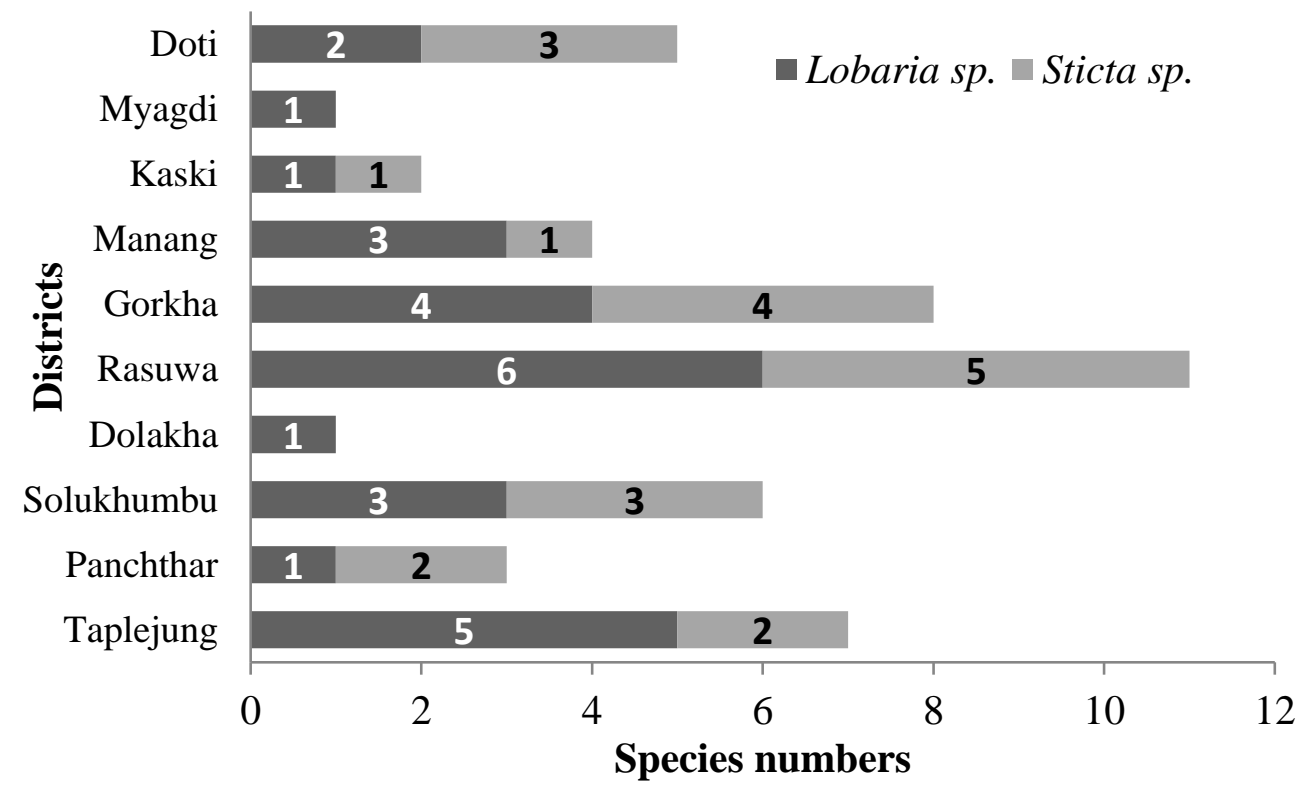

Fig. 2 - Total number of species of the genera Lobaria and Sticta from ten districts of Nepal. The order of the districts is arranged from East to West (Taplejung to Doti).

The altitudinal distribution range of the Lobariaceae of Nepal was determined on the basis of recent collections and earlier publications (Fig. 3). Most of the Lobariaceae species are distributed within the $2000 \mathrm{~m}$ to $4000 \mathrm{~m}$ altitudinal zone. This zone represents the temperate zone, where large variation in water availability occurs (Baniya et al. 2010). Sticta weigelii and Lobaria discolor are found in subtropical zone $(1000 \mathrm{~m}-2000 \mathrm{~m})$, L. retigera and L. pindarensis, are found in the alpine zone $(4000 \mathrm{~m}-5000 \mathrm{~m})$. Interestingly, one species, $L$. isidiosa, is found in the nival zone (above $5000 \mathrm{~m})$. The remaining species are found from the temperate to subalpine zone $(2000 \mathrm{~m}-4000 \mathrm{~m})$. L. isidiosa shows the largest altitudinal range $(2662 \mathrm{~m}-5004 \mathrm{~m})$, followed by L. retigera $(2141 \mathrm{~m}$ $4200 \mathrm{~m}$ ) and S. praetextata (2036 m - $3908 \mathrm{~m})$. Sticta limbata was found only in one location, Kyanjin valley (3180 m), Langtang National Park, Rasuwa District during the Langtang expedition of 2007.

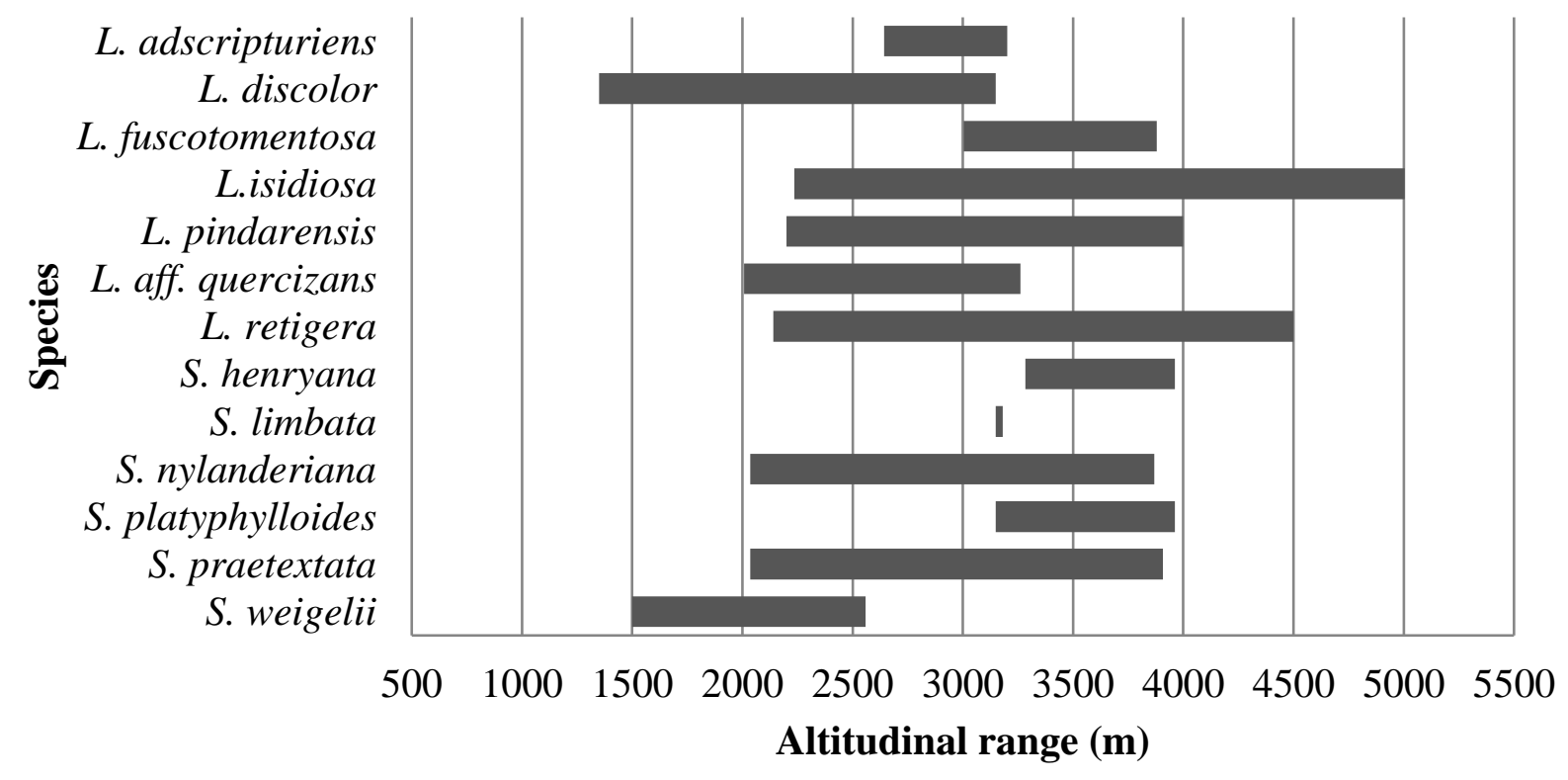

Fig. 3 - Distribution of Lobariaceae in different altitudinal levels, corresponding to bioclimatic zones. 
According to Shrestha (2008) the five bioclimatic zones in Nepal include the tropical zone (< $1000 \mathrm{~m})$, the sub-tropical zone $(1000 \mathrm{~m}-2000 \mathrm{~m})$, the subalpine zone $(3000 \mathrm{~m}-4000 \mathrm{~m})$, the alpine zone $(4000 \mathrm{~m}-5000 \mathrm{~m})$ and the nival zone $(>5000 \mathrm{~m})$. About two-thirds of the total forest area $(5.96$ million ha, i.e. $40.36 \%$ ) of Nepal is badly affected by grazing, tree cutting, bush cutting, looping, forest fire and other anthropogenic disturbances including bark removal from the trees (DFRS 2015). These kinds of disturbances decrease habitat quality and cause serious consequences for lichen, abundance and growth (Scheidegger \& Werth 2009).

\section{Taxonomic treatment}

\section{Identification keys to Lobaria and Sticta species in Nepal}

I. Cyphellae and pseudocyphellae on the lower side absent...................................................obaria

II. Cyphellae on the lower side present, medulla white.. Sticta

\section{Genus: Lobaria}

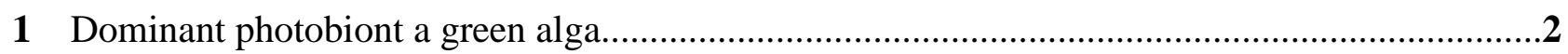

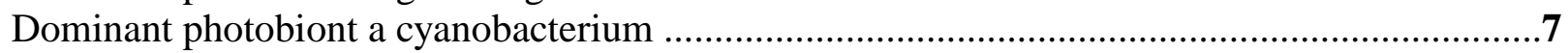

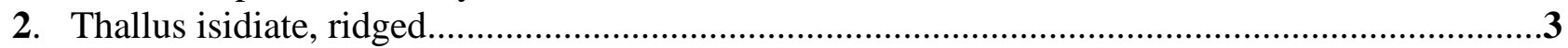

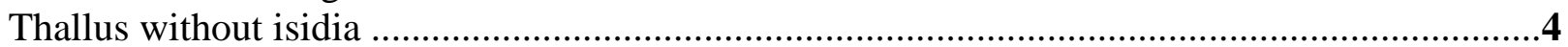

3. Medulla, $\mathrm{KC}+$, gyrophoric acid present................................................................ L. pindarensis

4. Medulla $\mathrm{KC}+$, ascospores fusiform, 1-3 septate, tomentum sparse............................... discolor Medulla $\mathrm{KC}+$, ascospores acicular, lower side dark brown to brown black...............................5

5. Cortex $\mathrm{K}+$, upper side wrinkled-rugose, gyrophoric and congygrophoric acid present

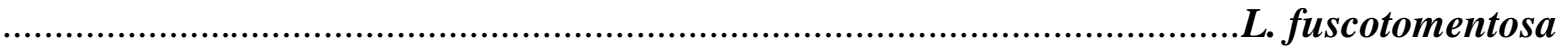

Cortex $\mathrm{K} \pm$, upper side smooth, medulla $\mathrm{KC}+$, gyrophoric acid present.

6. Cortex $\mathrm{K}-$, congyrophoric acid present, thallus dull greenish-brown L. adscripturiens Cortex $\mathrm{K}+$, congyrophoric acid absent, apothecia common, thallus greenish gray......

L. aff. quercizans

7. Retigeranic acid present, retigeric acid absent .L. isidiosa Retigeranic acid absent, retigeric acid present.

L. retigera

\section{Genus: Sticta}

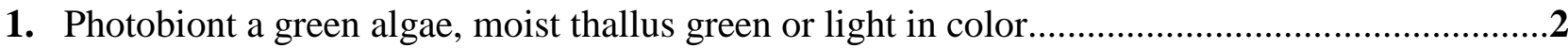
Photobiont blue - green algae (a cyanobacterium), moist thallus, dark......................................5

2. Thallus isidiate, medulla $\mathrm{KC}+$ Thallus lacking isidia and soredia.... S. praetextata

3. Medulla $\mathrm{KC}+$, gyrophoric acid present S. nylanderiana Medulla KC -, gyrophoric acid absent, thallus dichotomously lobed at margins......

4. Smooth thallus on upper side, S. henryana Thallus granulose tomentose on upper side. S. platyphylloides

5. Thallus lacking basal holdfast, attached by a single point, sorediate or isidiate

6. Thallus with densely sorediate margins, medulla $\mathrm{K}-$, S. limbata Margin with cylindrical isidia, medulla $\mathrm{K}+$

S. weigelii

A total of nine lichen substances were identified in the specimens examined by color reactions and thin layer chromatography. For species, whose specimens were not available, chemical properties were adapted from earlier publications (see details on enumeration). Gyrophoric acid, congyrophoric acid, atranorin, norstictic acid, stictic acid, constictic acid, thelophoric acid, retigeric acid, and retigeranic acid were identified from Lobaria and Sticta species (Table 1). 
Table 1 - Color reactions and TLC results of the Lobaria and Sticta species.

\begin{tabular}{|c|c|c|c|c|c|c|c|c|c|c|c|c|c|c|c|c|}
\hline \multirow[b]{2}{*}{ Species } & \multicolumn{3}{|c|}{ Cortex } & \multicolumn{4}{|c|}{ Medulla } & \multicolumn{7}{|c|}{ Lichen substances } & \multirow[b]{2}{*}{ R1 } & \multirow[b]{2}{*}{$\mathbf{R 2}$} \\
\hline & $\mathbf{K}$ & C & $\mathrm{KC}$ & $\mathbf{K}$ & $\mathbf{C}$ & KC & $\mathbf{P}$ & Gyr & Cong & Atr & Nor & Sti & Cons & Thel & & \\
\hline \multicolumn{17}{|c|}{ Lobaria (Schreb.) Hoffm., } \\
\hline L. adscripturiens & - & - & - & - & - & + & - & + & + & $?$ & - & - & - & - & - & - \\
\hline L. discolor & + & - & - & - & - & + & - & + & - & - & - & - & - & - & - & - \\
\hline L. fuscotomentosa & + & - & - & + & - & + & - & + & + & - & - & - & - & - & - & - \\
\hline L. isidiosa & - & - & - & \pm & - & - & - & - & - & - & + & + & + & - & - & + \\
\hline L. pindarensis & - & - & - & + & - & + & + & + & - & - & + & + & + & - & - & - \\
\hline L. aff. quercizans & + & - & - & - & - & + & - & + & - & - & - & - & - & - & - & - \\
\hline L. retigera & - & - & - & \pm & - & - & - & - & - & - & - & - & - & + & + & - \\
\hline
\end{tabular}

\section{Sticta (Schreb.) Ach.}

\begin{tabular}{|c|c|c|c|c|c|c|c|c|c|c|c|c|c|c|c|c|}
\hline S. henryana & - & - & - & - & - & - & - & - & - & - & - & - & - & - & - & - \\
\hline S. limbata & - & - & - & - & - & - & - & - & - & - & - & - & - & - & - & - \\
\hline S. nylanderiana & + & - & - & - & - & + & - & + & + & + & - & - & - & - & - & - \\
\hline S. platyphylloides & - & - & - & - & - & - & - & - & - & - & - & - & - & - & - & - \\
\hline S. praetextata & + & - & - & - & - & + & - & + & - & + & - & - & - & - & - & - \\
\hline S. weigelii & - & - & - & + & - & - & - & - & - & - & - & - & - & - & - & - \\
\hline
\end{tabular}

$\mathrm{K}=$ Potassium hydroxide $(\mathrm{KOH}) ; \mathrm{C}=$ Aqueous solution of calcium hypochlorite $(\mathrm{Ca}(\mathrm{OCl}) 2) ; \mathrm{P}=$ Aqueous solution of p-phenylenediamine $(\mathrm{C} 6 \mathrm{H} 4(\mathrm{NH} 2) 2)$; $\mathrm{Gyr}=$ gyrophoric acid; Cong = congyrophoric acid; Atr = atranorin; Nor = norstictic acid; Sti = stictic acid; Cons = constictic acid; Thel $=$ thelophoric acid; R1=Retigeric acid; R2=Retigeranic acid

Enumeration of species

Lobaria (Schreb.) Hoffm.,

Lobaria adscripturiens (Nyl.) Hue

Etymology - Thallus 6-12 cm broad, light-yellow brown, irregular and wide lobes $(0.5-1.0 \mathrm{~cm})$ lobes, wave shaped and shining margins, upper surface slightly wrinkled, soredia and isidia absent, green algae - photobiont, dark-brown tomentum, dark brown rhizines, pycnidia with black ostioles, dark brown colored and disc shaped apothecia $(0.5-3.5 \mathrm{~mm})$ in diameter, acicular, 3 septate spores $42-45$ $\times 5-7 \mu \mathrm{m}$.

Known distribution - India (Joshi \& Awasthi 1982, Awasthi 2007, Singh \& Sinha 2010), China, Taiwan, Philippines and Malaysia (Yoshimura 1971), South Korea (Park 1990, Ren et al. 2012).

New addition to Nepal.

Chemistry - Cortex $\mathrm{K}-, \mathrm{C}-, \mathrm{KC}-, \mathrm{P}-$; medulla $\mathrm{K}-, \mathrm{C}-, \mathrm{KC}+($ rose red), $\mathrm{P}-$. TLC: gyrophoric, congyrophoric and traces of atranorin present.

Number of specimens examined - 13

Material examined (Representative only) - RASUWA: Thulo Syafru, Gosainkund valley. Between Chandanbari to Gosaikund, on bark of Abies spectabilis $28^{\circ} 07.635^{`} \mathrm{~N}, 85^{\circ} 20.905^{`} \mathrm{E}$, alt. 3202 m, 27 September 2007, Joshi, Sharma \& Olley 2007, M2; Ghoda Tabela on the way to Kyanjin, on trunk of Quercus semecarpifolia, $28^{\circ} 11.838^{`} \mathrm{~N}, 85^{\circ} 27.242^{`}$ E, alt. $3067 \mathrm{~m}$, April 27, 2009. Scheidegger \& Devkota, 2009, NE69/06a; Lama Hotel on the way to Kyanjin, on trunk of Alnus nepalensis, $28^{\circ} 10.166^{`} \mathrm{~N}, 85^{\circ}$ 26. 176 E, alt. $2642 \mathrm{~m}$, April 27, 2009. Scheidegger \& Devkota, 2009, NE72/08b.

Distribution range in Nepal - Temperate - subalpine zone, C, $2642 \mathrm{~m}$ - 3202 m. Fig. 4A

Conservation Status - Critically endangered (CR)

Notes - This species superficially resembles L. quercizans but is distinguished by the presence of congyrophoric acid in the medulla, and its wrinkled or rough thallus. The systematic position of the specimens from Nepal needs to be confirmed with molecular analyses.

Lobaria discolor (Bory) Hue 
Known distribution - NEPAL: Mewakhola valley (1350 m), Eastern Nepal (Joshi \& Awasthi 1982); Khaptad (3150m), Doti district, Seti Zone (Sharma 1979b), India (Joshi \& Awasthi 1982); Philippines, Sri Lanka (Yoshimura 1971), South Korea (Ren et al. 2012).

Chemistry - Cortex $\mathrm{K}+$ (yellow), $\mathrm{C}-, \mathrm{KC}-, \mathrm{P}-$; medulla $\mathrm{K}-, \mathrm{C}-, \mathrm{KC}+$ (red), $\mathrm{P}-$. TLC: gyrophoric

Number of specimens examined - 1

Material examined - KASKI: Near Australian Camp, Lumle $28^{\circ} 18.381^{`} \mathrm{~N}, 83^{\circ} 50.087^{`} \mathrm{E}$, alt. 1980 m, April 27, 2014, Scheidegger, 2014. Nepal.

Distribution range in Nepal - Sub-tropical - subalpine zone, E, W, $1350 \mathrm{~m}$ - 3150 m. Fig. 4B Conservation Status - Vulnerable (VU)

Notes - The systematic position of the specimens from Nepal needs to be confirmed with molecular analyses.

Lobaria fuscotomentosa Yoshim.

Etymology - Thallus foliose, $7 \mathrm{~cm}$ long, dark brown, lobes $(6-12 \mathrm{~mm})$, wrinkled surface, green algae - photobiont, dark-brown tomentum, pycnidia $0.2-0.3 \mathrm{~mm}$ in diameter, soredia, isidia and cyphellae absent on both surfaces, dark brown colored apothecia (1.5-3.5 $\mathrm{mm}$ ) in diameter, acicular 2- septate spores 30-42 × 3-6 $\mu \mathrm{m}$.

Known distribution - India (Joshi \& Awasthi 1982, Pandit \& Sharma 2012); Bhutan (Aptroot \& Feijen 2002), Japan, China (Yoshimura 1971)

New addition to Nepal.

Chemistry - Cortex $\mathrm{K}+$ (yellow), $\mathrm{C}-, \mathrm{KC}-, \mathrm{P}-$; medulla $\mathrm{K}+$ (pale yellow), $\mathrm{C}-, \mathrm{KC}+$ (reddish), P -. TLC: gyrophoric, congyrophoric

Number of specimens examined - 3

Material examined - RASUWA: Kyanjin valley to Ghoda Tabela, on bark of Abies spectabilis $28^{\circ}$ 12.246 N, 85 35.519 E, alt. 3879 m, September 24, 2007, Olley, Cross, Joshi \& Sharma, 2007, AC11.1; GORKHA: Lho, Nubri valley on the way to Samagaun, on bark of Abies spectabilis, $28^{\circ}$ 34.258 N, 84 41.905 E, alt. 3284 m, 26 March 2011. Devkota, Sankhi \& Scheidegger 2011, 14WF1/26; TAPLEJUNG; Gyabla , Ghunsa valley, on the way to Ghunsa, Kangchenjunga Conservation Area, on bark of Quercus semicarpifolia, $27^{\circ} 37.323^{`} \mathrm{~N}, 87^{\circ} 52.548^{`} \mathrm{E}$, alt. $3003 \mathrm{~m}, 6$ April, 2012, Devkota, Chongbang \& Scheidegger 2012, 63WE1/33.

Distribution range in Nepal - Subalpine zone, E, C,W, $3003 \mathrm{~m}-3879$ m. Fig. 4C

Conservation Status - Vulnerable (VU)

Notes - The systematic position of the specimens from Nepal needs to be confirmed with molecular analyses.

Lobaria isidiosa (Müll. Arg.) Vain

Known distribution - NEPAL: Tato Pani - Marsyangdi river, Manang district(Asahina 1955); Rolwaling Himalaya, Dolakha (Kurokawa 1967); Cho-Oyu Himalaya near Debuche 3657 - 3962 m.(Awasthi 1960); Batassy Bhuspate Dandaa - Panchthar Yoshimura (1971); Mewakhola valley (3300 m - 3450 m) Taplejung - Eastern Nepal (Joshi \& Awasthi 1982); Langtang - Rasuwa (Miehe 1990), India (Joshi \& Awasthi 1982, Pinokiyo et al. 2008, Molleman et al. 2011, Joshi et al. 2016), Japan, China, Bhutan, Philippines and Indonesia (Yoshimura 1971), South Korea (Ren et al. 2012), Taiwan (Lin 2013), Alaska and British Columbia (Cornejo \& Scheidegger 2015)

Chemistry - Cortex $\mathrm{K}-, \mathrm{C}-, \mathrm{KC}-, \mathrm{P}-$; medulla $\mathrm{K} \pm$ (yellow), $\mathrm{C}-, \mathrm{KC}-, \mathrm{P}-$. TLC: norstictic, stictic, constictic and, retigeranic acid

Number of specimens examined - 119

Material examined (Representative only) - RASUWA: Kyanjin valley between Ghoda Tabela and Kyanjin, on bark of Sorbus microphylla, $28^{\circ} 12.246^{`}$ N, $85^{\circ} 33.31^{`}$ E, alt. 3835 m, September 24, 2007, Cross, Joshi, Sharma \& Olley, 2007, AC4.2; Tserko Ri above Kyanjin, Kyanjin valley on dwarf scrubs, $28^{\circ} 12.787^{`} \mathrm{~N}, 85^{\circ} 36.35^{`} \mathrm{E}$, alt. $5004 \mathrm{~m}$, April 23, 2009. Scheidegger \& Devkota, 2009. NE58/a. GORKHA: Samagaun, Samagaun valley, on bark of Betula utilis, $28^{\circ} 34.973^{`}$ N, 
$84^{\circ}$ 38.495 E, alt. 3501 m, March 27 2011. Devkota, Sankhi \& Scheidegger 2011, 15WE1/20; Dumje (Opposite Gho), Tsum valley, on trunk of Quercus semicarpifolia, $28^{\circ} 28.113^{`} \mathrm{~N}, 85^{\circ} 00.486^{`} \mathrm{E}$, alt. 2563 m, 19 September 2012. Devkota \& Scheidegger 2012, 42EF1/10. SOLUKHUMBU: Surkey, Dudhkoshi valley on the way to Lukla, on trunk of Gultheria fragmentissima, $27^{\circ} 40.328^{\prime} \mathrm{N}, 86^{\circ}$ 42.868` E, alt. 2209 m, July 04, 2012, Devkota \& Scheidegger 2012, 21EF2/11.Tongnasa, Dudhkunda valley on the way to Dudhkunda lake, on bark of Tsuga dumosa, $27^{\circ} 35.681^{\prime} \mathrm{N}, 86^{\circ}$ 35.379 E, alt. 2968 m, 18 June, 2012, Devkota \& Scheidegger 2012, 53WF1/5. TAPLEJUNG: Rambuk Kharka, Ghunsa valley above Ghunsa, on trunk of Juniperous indica, $27^{\circ} 42.813^{`} \mathrm{~N}, 87^{\circ}$ 57.919 E, alt. 3850 m, Aspect 120, Slope 30, 11 April, 2012, Devkota, Chongbang \& Scheidegger 2012, 65WF2/30.

Distribution range in Nepal - Temperate - nival zone, E, C, W, 2235 m - 5004 m. Fig. 4D In our study area, it is collected from $2235 \mathrm{~m}-3850 \mathrm{~m}$. Earlier, the highest range reported by Awasthi (1960) was 3962 m from Cho-Oyu Himalaya near Debuche.

Conservation Status - Least concern (LC)

Notes - Lobaria isidiosa looks like L. pindarensis, but it has Nostoc as a photobiont (Awasthi 2007). Cornejo et al. (2009) found the presence of retigeranic acids but absence of retigeric acid in $L$. isidiosa.

\section{Lobaria pindarensis Räsänen}

Known distribution - NEPAL: Mewakhola valley (3300 m) -Taplejung, Eastern Nepal (Joshi \& Awasthi 1982); Olangchung Gola - Taplejung (3400 m), Khumjung - Solukhumbu (3871m), Dumje forest - Tsum, Gorkha (3400 m) (Devkota et al. 2014), Bhutan (Upreti \& Ranjan 1988, Upreti \& Negi 1996, Aptroot \& Feijen 2002), India (Joshi \& Awasthi 1982, Shukla et al. 2015).

Number of specimens examined - 1391

Material examined (Representative only) - RASUWA: Ghoda Tabela, Gosaikunda valley, on branch of Berberis sp. near Army camp alt. 3100 m, Sept.19 2007, Joshi, Sharma \& Olley, 2007, M1. Between Gosaikunda and Chandanbari, Gosaikunda valley, on trunk of Abies spectabilis $28^{\circ}$ 05.718 N, 85 22.523` E, alt. 3765 m, April.19 2009, Scheidegger \& Devkota, 2009, NE37/02a. Lama Hotel, Kyanjin valley $28^{\circ} 09.458^{`}$ N, 85 25.19` E, alt. 2036 m, 21 April 2009. Scheidegger \& Devkota 2009, NE56/01a. Kyanjin, Kyanjin valley $28^{\circ} 12.458^{`}$ N, $85^{\circ} 33.19^{`}$ E, alt. 3884 m, 21 April 2007. Cross, Joshi, Sharma \& Olley 2007, AC10. GORKHA: Gaup, Nubri valley on the way to Samagaun, on trunk of Rhus wallichi, $28^{\circ} 31.927^{`}$ N, 84 48. $787^{`}$ E, alt. 2200 m, March 31, 2011. Devkota \& Scheidegger, 2011. MP15/01a. Samagaun, Nubri valley, on trunk of Abies spectabilis, $28^{\circ} 34.406^{`} \mathrm{~N}, 84^{\circ} 40.075^{`}$ E, alt. $3570 \mathrm{~m}$, March 29, 2011. Devkota \& Scheidegger, 2011. MP1/20a. Dumje (Opposite Gho), Tsum valley, on trunk of Rhus wallichi, $28^{\circ} 28.28^{`} \mathrm{~N}, 85^{\circ} 00.771^{`} \mathrm{E}$, alt. $2600 \mathrm{~m}$, September 20, 2012. Devkota \& Scheidegger, 2012.M2P1/01a. Lamjung (Opposite Chekampar) Tsum valley, on branch of Rosa macrophylla, $28^{\circ} 28.514^{`} \mathrm{~N}, 85^{\circ} 03.131^{`} \mathrm{E}$, alt. 3800 $\mathrm{m}$, September 24, 2012. Devkota \& Scheidegger, 2012.M2P9/01a. SOLUKHUMBU: Musey, Dudhkoshi valley on branch of Berberis sp, $27^{\circ} 41.614^{`} \mathrm{~N}, 86^{\circ} 43.094 \mathrm{E}$, alt. $2569 \mathrm{~m}$, April 13, 2011, Devkota \& Scheidegger, 2011. 22EE1/11; Khumjung, Dudhkoshi valley, on trunk of Abies spectabilis, $27^{\circ} 48.871^{`} \mathrm{~N}, 86^{\circ} 43.016$ E’, alt. $3871 \mathrm{~m}$, April 18, 2011, Devkota \& Scheidegger, 2011. SP1/35a; Phera, Dudhkunda valley on trunk of Pinus wallichiana, $27^{\circ} 33.869^{\circ} \mathrm{N}, 86^{\circ} 35$. 590` E, alt. 2600 m, June 15 2012, Devkota \& Scheidegger, 2012. 52EE2/20; Mangem, Dudhkunda valley on trunk of Abies spectabilis, $27^{\circ} 39.370^{\prime} \mathrm{N}, 86^{\circ} 35.803 \mathrm{E}^{\prime}$, alt. $3795 \mathrm{~m}$, June 24, 2012, Devkota \& Scheidegger, 2012. 55EE2/1. TAPLEJUNG: Gyabla, Ghunsa valley, on Arundinaria sp, $27^{\circ}$ 36. $675^{`}$ N, $87^{\circ}$ 52. 333 E`, alt. 2486 m, April 13 2012, Devkota \& Scheidegger, 2012. K2P9/01a. Rambuk Kharka, Ghunsa valley, on trunk of Juniperous indica sp, $27^{\circ} 42.791^{`} \mathrm{~N}, 87^{\circ}$ 57. 871E', alt. 3845 m, April 11, 2012, Devkota, Chongbang \& Scheidegger, 2012. 65WE2/2. Olangchung Gola (opposite forest), Olangchung Gola valley, on bark of Viburnum nervosum, $27^{\circ}$ 40. 306` N, $87^{\circ}$ 46. 836 E`, alt. 3167 m, May 16, 2011, Devkota \& Scheidegger, 2011. 33WF1/04. Nearby Dinga Samba, Olangchung Gola valley, on branch of Rhodendron campanulatum, $27^{\circ} 43$. $010^{`} \mathrm{~N}, 87^{\circ}$ 43. $998^{`} \mathrm{E}$, alt. $4000 \mathrm{~m}$, May 20, 2011, Devkota \& Scheidegger, 2011. KP2/20a. 
MANANG: Bhimtang, on trunk of Abies spectabilis, $28^{\circ} 37.338^{`} \mathrm{~N}, 84^{\circ} 28.091 \mathrm{E}^{`}$, alt. $3600 \mathrm{~m}$, October 16, 2012, Devkota \& Scheidegger, 2012. MAP1 / 21b. Kharche, Surki Khola, below Bhimtang on trunk of Mahonia nepalensis alt. $2681 \mathrm{~m}$, October 16, 2012, Devkota \& Scheidegger, 2012. Between Bhratang and Chame $28^{\circ} 33.996^{`} \mathrm{~N}, 84^{\circ} 12.663^{`} \mathrm{E}$, alt. $2761 \mathrm{~m}$, April 13, 2014, Scheidegger, 2014. CS982. Above Pisang, $28^{\circ} 36.453^{`} \mathrm{~N}, 84^{\circ} 08.839^{`}$ E, alt. $3461 \mathrm{~m}$, April 14, 2014, Scheidegger, 2014. C99-Nepal. MYAGDI: Ghodepani $28^{\circ} 24.598^{`}$ N, $84^{\circ} 41.907^{`}$ E, alt. 2659 m, April 23, 2014, Scheidegger, 2014. K99-Nepal. Deurali near Ghodepani $28^{\circ} 24$.

Chemistry - Cortex $\mathrm{K}-, \mathrm{C}-, \mathrm{KC}-, \mathrm{P}-$; medulla $\mathrm{K}+$ (yellow) $\mathrm{C}-, \mathrm{KC}+$ (red), $\mathrm{P}+$ (yellow). TLC: gyrophoric, norstictic, stictic and constictic

Distribution range in Nepal - Temperate - Subalpine zone, E, C, W, $2036 \mathrm{~m}$ - 4000 m. Fig. 4E Conservation status - Vulnerable (VU)

Notes - Lobaria pindarensis is associated with the green algal photobiont Symbiochloris reticulata (Skaloud et al. 2016). This species has been described from different geographical locations of the Himalayas. Yoshimura (1969) treated this species under L. meridionalis, but the later one is distinguished by the absence of gyrophoric acid and it is likely that he made a misidentification. Similarly, Joshi \& Awasthi (1982) reported L. meridionalis from Mewakhola valley (3300m), Taplejung Nepal but it is likely that they made a wrong identification of $L$. pindarensis.

Miehe (1990) reported Lobaria pulmonaria from the Langtang territory of Nepal, but that could be Lobaria pindarensis. During 2007 and 2009 explorations, we observed several localities with a large population of $L$. pindarensis. Similar unconfirmed reports of $L$. pulmonaria were made by Smith (1931) from Darjeeling, and Singh (1981) from Manipur, India (Awasthi 2007).

Lobaria aff. quercizans Michx.

Etymology - Thallus corticolous, 7-12 cm across, pale brown, lobes $(5-11 \mathrm{~mm})$, plane surface and often wrinkled in older parts, greener when wet, green algae - photobiont, soredia and isidia absent, diffuse type tomenta, reddish-brown colored apothecia $(3-5 \mathrm{~mm})$ in diameter, acicular 3- septate spores $30-75 \times 4.5-7.5 \mu \mathrm{m}$.

Known distribution - India (Joshi \& Awasthi 1982); Bhutan (Aptroot \& Feijen 2002); Taiwan (Lin 2013), Japan, Russia - Sakhalin (Yoshimura 1971), Canada (Edman et al. 2008), South Korea (Ren et al. 2012).

New addition to Nepal.

Chemistry - Cortex $\mathrm{K}+$ (yellow), $\mathrm{C}-, \mathrm{KC}-, \mathrm{P}-$; medulla $\mathrm{K}-, \mathrm{C}-, \mathrm{KC}+$ (pink), $\mathrm{P}-$. TLC: gyrophoric

Number of specimens examined - 7

Material examined (Representative only) - RASUWA: Thulo Syafru, Gosainkunda valley on the way to Gosaikunda, on branch of Sorbus microphylla, $28^{\circ} 07.174 ` \mathrm{~N}, 85^{\circ} 20.435^{`} \mathrm{E}$, alt. $3319 \mathrm{~m}$, April 20, 2009. Scheidegger \& Devkota, 2009. NE48 / 01a; Lama Hotel, Kyanjin valley, on the way to Kyanjin, on trunk of Abies spectabilis, $28^{\circ} 09.314^{`} \mathrm{~N}, 8^{\circ} 23.850^{`} \mathrm{E}$, alt. $2007 \mathrm{~m}$, April 21, 2009. Scheidegger \& Devkota, 2009. NE55 / 01b.

Distribution range in Nepal - Temperate - Subalpine zone, C, 2007 m - 3262 m. Fig. 4A

Conservation status - Data deficient (DC)

Notes - The systematic position of the specimens from Nepal needs to be confirmed with molecular analyses.

\section{Lobaria retigera (Bory) Trevis.}

Known distribution - NEPAL: Laskya La (4200m) and Tolo Gompa Khola (4100m) - Manaslu (Asahina 1955); Bilbatay Bhanjyang, Tapethok - Helok, Bayoya - Helok, Khimty, Taplejung (Yoshimura 1971); On the way to Khaptad near herbal farm (3150 m), Doti (Sharma 1979b); Rakshe - Ethung, Taplejung (2250 m), Mewakhola valley, Tankhu village, Topkegola - Thaglabhangang, Taplejung (2250 m - 4500 m) Eastern Nepal (Joshi \& Awasthi 1982), Langtang - Rasuwa (Miehe 1990). Bhutan (Upreti \& Ranjan 1988, Søchting 1999, Aptroot \& Feijen 2002), India (Joshi \& Awasthi 1982, Pinokiyo et al. 2008, Shukla et al. 2015, Joshi et al. 2016), Japan, China, Vietnam, 
Thailand, Philippines, New Guinea (Yoshimura 1971), Taiwan (Lin 2013), Alaska (Jordan 1973, Geiser et al. 1994)

Chemistry - Cortex $\mathrm{K}-, \mathrm{C}-, \mathrm{KC}-, \mathrm{P}-$; medulla $\mathrm{K} \pm, \mathrm{C}-, \mathrm{KC}-, \mathrm{P}-$. TLC: thelophoric, triterpenoid and, retigeric acid.

Number of specimens examined - 397

Material examined (Representative only) - RASUWA: Kyanjin, on rock, $28^{\circ} 12.217^{\circ} \mathrm{N}, 85^{\circ} 33$. 417 E, alt. 3869 m, April 24, 2009. Joshi, Sharma \& Olley, 2007. M2.3. GORKHA: Gaup, Nubri valley on the way to Samagaun, on trunk of Machelus sp, $28^{\circ} 32.132^{`} \mathrm{~N}, 84^{\circ} 48.117^{`} \mathrm{E}$, alt. 2147 m, March 20, 2011. Devkota, Sankhi \& Scheidegger, 2011. 11WF1/09; Samagaun, Nubri valley on mossy rock, $28^{\circ} 34.420^{`} \mathrm{~N}, 84^{\circ} 39.672^{`} \mathrm{E}$, alt. $3570 \mathrm{~m}$, March 29, 2011. Devkota, \& Scheidegger, 2011. MP1/09a. Lokpa, Tsum valley, on mossy rock, $28^{\circ} 26.352^{`} \mathrm{~N}, 84^{\circ} 54.799^{`}$ E, alt. $2179 \mathrm{~m}$, September 13, 2012, Devkota \& Scheidegger, 2012. 41EE1/01. Chekampar, Tsum valley, on trunk of Abies spectabilis, $28^{\circ} 28.793^{`} \mathrm{~N}, 85^{\circ} 02.974^{`} \mathrm{E}$, alt. $3400 \mathrm{~m}$, September 24, 2012, Devkota \& Scheidegger, 2012. M2P7/01b. TAPLEJUNG: Olangchung Gola on branch of Berberis sp, $27^{\circ} 40$. $399^{`} \mathrm{~N}, 87^{\circ} 46.771^{`} \mathrm{E}$, alt. 3157 m, May 16, 2011, Devkota \& Scheidegger, 2011.33WM1/08. Near Dinga Samba, Olangchung valley, on branch of Betula utilis, $27^{\circ} 43.012^{`} \mathrm{~N}, 87^{\circ} 43.913^{`} \mathrm{E}$, alt. 4000 m, May 20, 2011, Devkota \& Scheidegger, 2011. K2P2/02b. Sekathum, Ghunsa valley, on mossy rock, $27^{\circ} 32.156^{`} \mathrm{~N}, 87^{\circ} 48.956^{`} \mathrm{E}$, alt. $2184 \mathrm{~m}$, April 04, 2012, Devkota, Chongbang \& Scheidegger, 2012. 61EE1/16. Rambuk Kharka, Ghunsa valley, on branch of Salix sp, $27^{\circ} 42.550^{\circ}$ N, 87 57. 797 E, alt. 3791 m, April 11, 2012, Devkota, Chongbang \& Scheidegger, 2012. 65WE1/16. SOLUKHUMBU: Khumjung, Dudhkoshi valley, on trunk of Rhododendron campanulatum, $27^{\circ} 48.952^{`} \mathrm{~N}, 86^{\circ} 42.912^{`} \mathrm{E}$, alt. $3824 \mathrm{~m}$, July 11, 2012, Devkota \& Scheidegger, 2012. 25WE2/5; Musey, Dudhkoshi valley, on trunk of Quercus semicarpifolia, $27^{\circ} 40.903^{`} \mathrm{~N}, 86^{\circ}$ 42. 756 E, alt. 2212m, July 14, 2011, Devkota, Sankhi \& Scheidegger, 2011. 22EF1/18. Boldok, Dudhkunda valley, on rock, $27^{\circ} 31.130^{`} \mathrm{~N}, 86^{\circ} 34.442^{`} \mathrm{E}$, alt. $2176 \mathrm{~m}$, June 10, 2012, Devkota \& Scheidegger, 2012. 51WM2/01. Simidanda, opposite of Phera, Dudhkunda valley, on branch of Viburnum erubescense, $27^{\circ} 35.395^{`} \mathrm{~N}, 86^{\circ} 35.877^{`} \mathrm{E}$, alt. $2800 \mathrm{~m}$, June 16, 2012, Devkota \& Scheidegger, 2012. S2P2/24b. MANANG: Bhimtang, on trunk of Abies spectabilis, $28^{\circ} 37.338^{`} \mathrm{~N}$, $84^{\circ}$ 28. 091 E, alt. 3600 m, October 16, 2012, Devkota \& Scheidegger, 2012. MAP1 / 21b.

Distribution range in Nepal - Temperate - alpine zone, E, C, W, $2141 \mathrm{~m}$ - $4500 \mathrm{~m}$. Fig. 4F

In our study area, it is collected from $2141 \mathrm{~m}-4000 \mathrm{~m}$. An earlier report by Joshi \& Awasthi (1982) was from 2700 m - 4500 m from Thanglabhangang, Taplejung, Eastern Nepal.

Conservation status - Least concern (LC)

Notes - Cornejo et al. (2009) found the presence of retigeric acids and absence of retigeranic acids in $L$. retigera.

Sticta (Schreb.) Ach.

The species listed under the genus Sticta need a careful taxonomic revision. We assume that a detailed revision will lead to a substantial reorganization of the species complex around $S$. nylanderiana.

Sticta henryana Müll. Arg.

Known distribution - NEPAL: Near Debuche (3657 - 3962 m) Cho-Oyu Himalaya - Solukhumbu (Awasthi 1960). India (Joshi \& Awasthi 1982, Awasthi 2007, Singh \& Sinha 2010), Bhutan (Upreti \& Ranjan 1988, Aptroot \& Feijen 2002), China (Li et al. 1991).

Chemistry - Cortex $\mathrm{K}-, \mathrm{C}-, \mathrm{KC}-, \mathrm{P}-$; medulla $\mathrm{K}-, \mathrm{C}-, \mathrm{KC}-, \mathrm{P}-$. TLC: No lichen products present.

Number of specimens examined - 1

Material examined - GORKHA: Lho, Nubri valley, on bark of Abies spectabilis, $28^{\circ} 34.258^{`} \mathrm{~N}$, $84^{\circ} 41.905^{`}$ E, alt. 3284 m, 26 March 2011. Devkota, Sankhi \& Scheidegger 2011, 14WF1/25.

Distribution range in Nepal - Subalpine zone, C, W, $3284 \mathrm{~m}-3962 \mathrm{~m}$. Fig. 5A

In our study area, it is collected from $3284 \mathrm{~m}$. Earlier highest range was reported by Awasthi (1960) from Cho-Oyu Himalaya area (3962 m). 


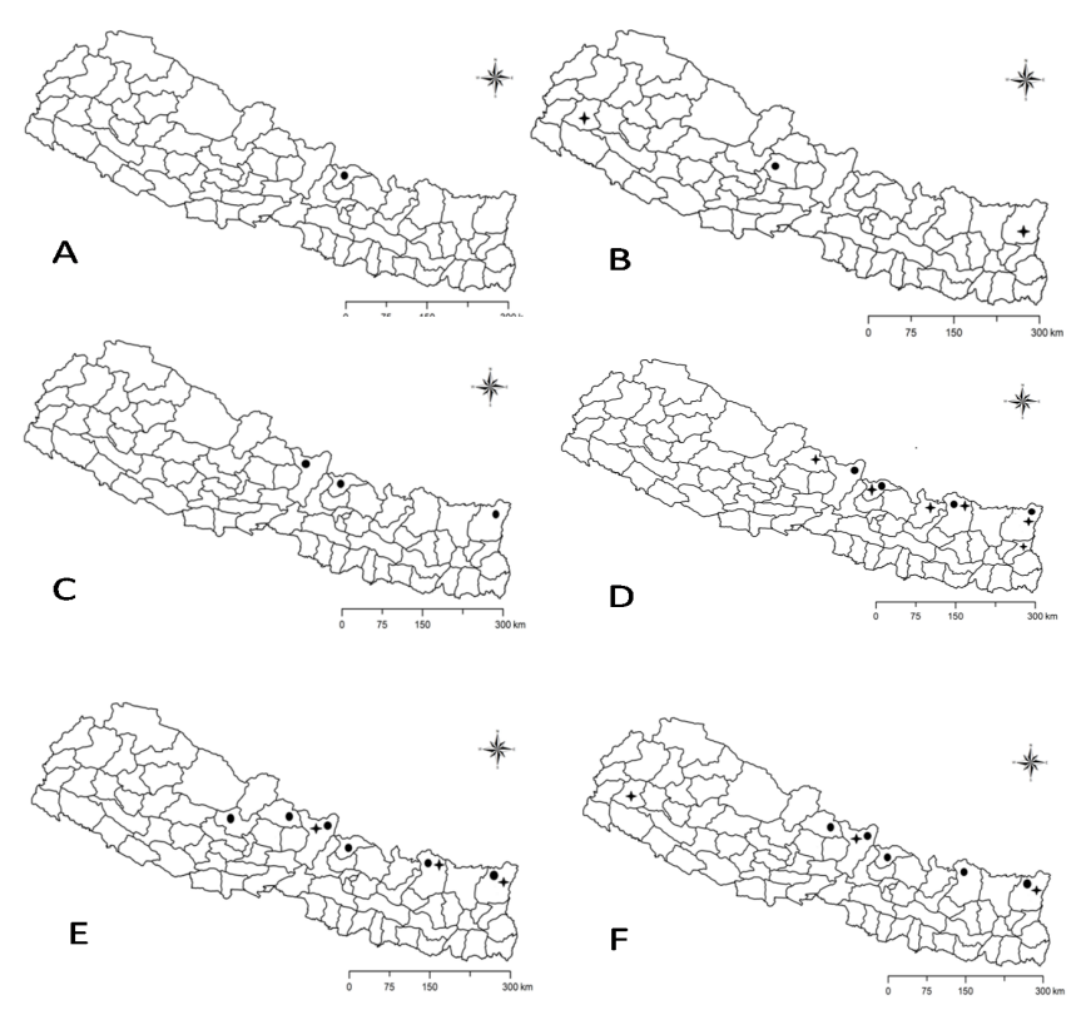

Fig. 4 - Distribution of Lobaria species in Nepal: A (L. adscripturiens and Lobaria aff. quercizans), $\mathbf{B}$ (L. discolor), $\mathbf{C}$ (L. fuscotomentosa), $\mathbf{D}$ (L. isidiosa), $\mathbf{E}$ (L. pindarensis), $\mathbf{F}$ (L. retigera). Symbols $(\bullet)$ and $(+)$ represent recently collected districts by the authors and previously reported districts respectively.

Conservation status - Endangered (EN)

Notes - The systematic position of the specimens from Nepal needs to be confirmed with molecular analyses.

Sticta limbata (Sm.) Ach.

Etymology - Thallus foliose, monophyllous, $5 \mathrm{~cm}$ long, yellowish grey, loosely attached at one point, lobes $0.5-2.5 \mathrm{~cm}$ in diameter and irregular, smooth and shining surface, marginally erose - soralia, without isidia, blue green algae - photobiont, lower surface - grayish brown, densely and wooly tomentum, apothecia not seen.

Known distribution - Bhutan (Aptroot \& Feijen 2002), British Columbia, Canada (Radies et al. 2009), Chile (Galloway 1994), India (Joshi \& Awasthi 1982, Awasthi 2007, Singh \& Sinha 2010), New Zealand (Oliver 1912), South Korea (Park 1990, Jayalal et al. 2014), UK (Ellis \& Hope 2012), USA (Neitlich \& Mccune 1997, McDonald et al. 2003), China (Wei 1991, Jinong \& Huajie 2012).

New addition to Nepal.

Chemistry - No lichen substances. TLC: No lichen products present.

Number of specimens examined - 1

Material examined - RASUWA: Ghoda Tabela to Langtang village, Kyanjin valley, on hard rock, $28^{\circ} 12.477^{`} \mathrm{~N}, 85^{\circ}$ 28. $658^{`}$ E, alt. 3180 m, September 19, 2009. Joshi, Sharma \& Olley, 2007. M3.1 Distribution range in Nepal - Subalpine zone, C, 3180 m. Fig. 5B

Conservation status - Critically endangered (CR)

Sticta nylanderiana Zahlbr.

Known distribution - NEPAL: Southern Mountain range of Annapurna 2500 m -3000 m, Kaski district (Asahina 1955); On way to Sahastraling, Khaptad near herbal farm - Doti (3015 m) (Sharma 
1979b), India (Awasthi 1965, 2007, Joshi \& Awasthi 1982, Singh \& Sinha 2010, Joshi et al. 2016), Bhutan (Upreti \& Ranjan 1988, Aptroot \& Feijen 2002), South Korea (Kim 1981, Jayalal et al. 2014). Chemistry - Cortex $\mathrm{K}+$ (yellow), $\mathrm{C}-, \mathrm{KC}-, \mathrm{P}-$; medulla $\mathrm{K}-, \mathrm{C}-, \mathrm{KC}+$ (pink), $\mathrm{P}-$. TLC: gyrophoric, congyrophoric, atranorin

Number of specimens examined - 289

Material examined (Representative only) - RASUWA: Gosaikunda, on trunk of Tsuga dumosa, $28^{\circ}$ 00. 550` N, $85^{\circ} 29.983^{`}$ E, alt. 3319 m, April 16, 2009. Scheidegger \& Devkota, 2009. NE15 / 01b; Kyanjin, on trunk of Alnus nepalense, $28^{\circ} 10.165^{`} \mathrm{~N}, 85^{\circ} 26.175^{`}$ E, alt. $2642 \mathrm{~m}$, April 27, 2009. Scheidegger \& Devkota, 2009. NE72 / 03K; GORKHA: Sho, Nubri valley, on the way to Samagaun, on branch of Tsuga dumosa, $28^{\circ} 34.109^{`}$ N, 84 43.301` E, alt. 2989 m, March 24, 2011. Devkota, Sankhi \& Scheidegger, 2011. 13WF1/24; Chekampar, Tsum valley, on trunk of Abies spectabilis, $28^{\circ}$ 28. $9835^{`} \mathrm{~N}, 85^{\circ} 02.817^{`} \mathrm{E}$, alt. $3060 \mathrm{~m}$, September 23, 2012, Devkota \& Scheidegger, 2012. 43EE1/02. TAPLEJUNG: Near Dinga Samba, Olangchung valley, on branch of Berberis aristata, $27^{\circ} 41.592^{`} \mathrm{~N}, 87^{\circ} 45.674^{`} \mathrm{E}$, alt. $3406 \mathrm{~m}$, August 21, 2011, Devkota \& Scheidegger, 2011. 34EM2 / 28. Rambuk Kharka, Ghunsa valley, on branch of Rhododendron campanulatum, $27^{\circ} 42.486^{\circ} \mathrm{N}, 87^{\circ}$ 57. $889^{`} \mathrm{E}$, alt. $3813 \mathrm{~m}$, April 12, 2012, Devkota, Chongbang \& Scheidegger, 2011. 34EM2 / 28. SOLUKHUMBU: Opposite of Musae, Dudhkoshi valley, on branch of Viburnu sp, $27^{\circ}$ 40. 498 N, 86 41. 949E', alt. 2806 m, April 23, 2011, Devkota \& Scheidegger, 2011. SP7 / 02a; Khumjung, Dudhkoshi valley, on trunk of Rhododendron campanulatum $27^{\circ} 48.952 ` \mathrm{~N}, 86^{\circ} 42.912 \mathrm{E}^{\prime}$, alt. $3824 \mathrm{~m}$, July 11, 2012, Devkota \& Scheidegger, 2012. 25WE2/4.Tongnasa, Dudhkunda valley, on bark of Tsuga dumosa $27^{\circ} 35.681^{\prime} \mathrm{N}, 86^{\circ} 35$. 379E', alt. 2968 m, June 18, 2012, Devkota \& Scheidegger, 2012. 55WF1/4. Sasarbeni, Dudhkunda valley, on branch of Juniperous sp., $27^{\circ} 39.373^{\prime} \mathrm{N}, 86^{\circ} 35.605 \mathrm{E}^{\prime}$, alt. $3777 \mathrm{~m}$, June 23, 2012, Devkota \& Scheidegger, 2012. 55WF2/3. MANANG: Bhimtang, on trunk of Abies spectabilis, $28^{\circ}$ 37. $338^{`} \mathrm{~N}, 84^{\circ} 28.091^{`} \mathrm{E}$, alt. $3600 \mathrm{~m}$, October 16, 2012, Devkota \& Scheidegger, 2012. MAP1 / $05 \mathrm{a}$.

Distribution range in Nepal - Temperate - subalpine zone, E, C, W, $2036 \mathrm{~m}$ - $3868 \mathrm{~m}$. Fig. 5C Conservation status - Least concern (LC)

\section{Sticta platyphylloides Nyl.}

Known distribution - NEPAL: Khaptad near herbal farm (3150 m) - Doti (Sharma 1979b), Near Debuche (3657 - 3962 m) Cho-Oyu Himalaya - Solukhumbu Awasthi (1960), India (Joshi \& Awasthi 1982, Awasthi 2007, Singh \& Sinha 2010, Joshi et al. 2016), Bhutan (Upreti \& Ranjan 1988, Søchting 1999, Aptroot \& Feijen 2002).

Chemistry - Cortex $\mathrm{K}-, \mathrm{C}-, \mathrm{KC}-, \mathrm{P}-$; medulla $\mathrm{K}-, \mathrm{C}-, \mathrm{KC}-, \mathrm{P}-$. TLC: None

Number of specimens examined - 19

Material examined (Representative only) - RASUWA: Chandanbari, Gosaikunda valley, on trunk of Abies spectabilis, $28^{\circ} 07.670^{`} \mathrm{~N}, 85^{\circ} 20.582^{`}$ E, alt. $3868 \mathrm{~m}$, April 19, 2009. Scheidegger \& Devkota, 2009. NE36 / 01i; Thulo Syafru, on trunk of Abies spectabilis, 28 07. 659 ${ }^{`}$ N, $85^{\circ} 20$. 870` E, alt. 3175 m, April 20, 2009. Scheidegger \& Devkota, 2009. NE49 / 01q.

Distribution range in Nepal - Subalpine zone, E, C, W, 3150 m - 3962 m. Fig. 5D

In our study area, it is collected from $3175 \mathrm{~m}-3868 \mathrm{~m}$. Earlier, the lowest range was reported by Sharma (1979a) from Khaptad Doti (3150 m) and the highest range was reported by Awasthi (1960) from Cho- Oyu Himalaya area (3962 m).

Conservation status - Vulnerable (VU)

Sticta praetextata (Räsänen) D.D.Awasthi

Known distribution - NEPAL: On way to Khaptad (2100 m) and near herbal farm (3150 m), Doti (Sharma 1979b), India (Joshi \& Awasthi 1982, Awasthi 2007, Singh \& Sinha 2010, Joshi et al. 2016), Bhutan (Søchting 1999; Aptroot \& Feijen 2002).

Chemistry - Thallus $\mathrm{K}+$ (yellow), $\mathrm{C}-, \mathrm{KC}-, \mathrm{P}-$; medulla $\mathrm{K}-, \mathrm{C}-, \mathrm{KC}+$ (pink), $\mathrm{P}-$. TLC: Gyrophoric, atranorin and unknown substances 


\section{Number of specimens examined - 57}

Material examined (Representative only) - RASUWA: Kyanjin, on trunk of Betula utilis, $28^{\circ} 12$. $210^{`} \mathrm{~N}, 85^{\circ} 33.800 \mathrm{E}$, alt. $3908 \mathrm{~m}$, September 21, 2007. Cross, Joshi, Sharma \& Olley, 2007. AC16. Kyanjin, on bark of Betula utilis, $28^{\circ} 12.254^{`} \mathrm{~N}, 85^{\circ} 33.452 \mathrm{E}$, alt. $3832 \mathrm{~m}$, April 25, 2009. Scheidegger \& Devkota, 2009. NE64 / 01K; Ghoda Tabela. GORKHA: Samagaun, Nubri valley, trunk of Abies spectabilis, $28^{\circ} 34.246^{`} \mathrm{~N}, 84^{\circ} 43.005^{`} \mathrm{E}$, alt. $3570 \mathrm{~m}$, March 29, 2011. Devkota \& Scheidegger, 2011. MP1/02b; Gaup, Nubri valley, on the way to Samagaun, on branch of Betula utilis, $27^{\circ}$ 48. 871 $\mathrm{N}, 86^{\circ}$ 43. 016 E, alt. $2400 \mathrm{~m}$, March 31, 2011. Devkota, Sankhi \& Scheidegger, 2011. MP14/16f; Chekampar, Tsum valley, on trunk of Tsuga dumosa, $28^{\circ} 28.983^{`} \mathrm{~N}, 85^{\circ} 02.817^{\circ}$ E, alt. 3060 m, September 23, 2012, Devkota \& Scheidegger, 2012. 43EE1/03. TAPLEJUNG Gyabla, Ghunsa valley, on trunk of Quercus semicarpifolia, $27^{\circ} 37.331^{`}$ N, $87^{\circ} 52.639^{`}$ E, alt. 3034 m, April 06, 2012, Devkota, Chongbang \& Scheidegger, 2012. 63WF1/01.

Distribution range in Nepal - Temperate - Subalpine zone, E, C, W, 2036 m - 3908 m. Fig. 5E Conservation status - Least concerned (LC)

Sticta weigelii (Ach.) Vain.

Known distribution - NEPAL: Mewakhola valley, near Shyamba village (1500 m), Taplejung, Eastern Nepal (Joshi \& Awasthi 1982), Bhutan (Aptroot \& Feijen 2002), India (Joshi \& Awasthi 1982), South Korea (Jayalal et al. 2014), Alaska (Geiser et al. 1994), Argentina and Chile (Galloway 1994), Malysia (Din et al. 2008).

Chemistry - Thallus $\mathrm{K}-\mathrm{C}-, \mathrm{KC}-, \mathrm{P}-$; medulla $\mathrm{K}+$ (yellow), $\mathrm{C}-, \mathrm{KC}-, \mathrm{P}-$. TLC: Unknown substances

Number of specimens examined - 2

Material examined - RASUWA: Thulo Syafru, Gosaikunda valley, on the way to Gosaikunda, on rock, $28^{\circ}$ 8. 434 N, 85 22. 239 E, alt. 2000 m, September 16, 2007. Olley, Joshi and Sharma 2007. L18. TAPLEJUNG: Sotlima, Ghunsa valley, Mathillo Tal, on the way to Ghunsa, on trunk of Quercus laminosa, $27^{\circ} 32.502^{`} \mathrm{~N}, 87^{\circ} 50.391^{`} \mathrm{E}$, alt. 2559 m, 16 April, 2012, Devkota, Chongbang \& Scheidegger 2012, 62EF1/08.

Distribution range in Nepal - Sub-tropical - temperate zone, E, C. $1500 \mathrm{~m}$ - $2559 \mathrm{~m}$. Fig. 5F In our study area, it is collected from $2000 \mathrm{~m}-2559 \mathrm{~m}$. Earlier, lowest range was reported by Joshi \& Awasthi (1982) from Mewakhola valley (1500 m).

Conservation status - Endangered (EN)

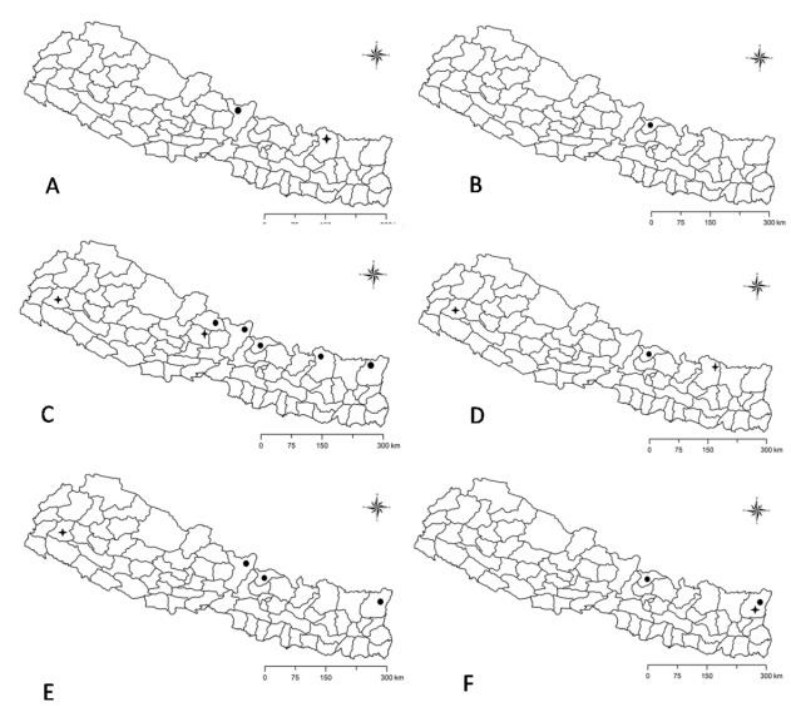

Fig. 5 - Distribution of Sticta species in Nepal: A (Sticta henryana), B (S. limbata), C $(S$. nylanderiana), D $(S$. platyphylloides $), \mathbf{E}(S$. praetextata), F (Sticta weigelii). Symbols $(\bullet)$ and $(+)$ represent recently collected districts by the authors and previously reported districts, respectively. 


\section{Acknowledgements}

We would like to thank the Central Department of Botany (CDB), Tribhuvan University (TU), and the Department of Plant Resources (DPR), Nepal for their cooperation. Department of National Parks and Wildlife Conservation (DNPWC), Ministry of Forest and Soil Conservation (MOFSC), Government of Nepal and the National Trust for Nature Conservation (NTNC) are thanked for providing research permission. Prof. Krishna Kumar Shrestha CDB and Carolina Cornejo, WSL is thanked for fruitful discussions. Many thanks to Sanjeev Kumar Rai, Jyoti Prasad Gajurel, Laxmi Sankhi, Til Bikram Chongbang, Hem Bahadur Katuwal, Lokendra Raj Sharma, Andrew Cross and Mohan Joshi for their support during field days. Sanjeeva Nayaka from National Botanical Research Institute, India is thankful for providing scanned copies of old literature from India. This research was funded by The Swiss National Science Foundation, Switzerland (grant JRP IZ70Z0_131338 / 1) to C. Scheidegger and Davis Expedition fund, Cryptogamic work group, Sibbald Trust, Percy Sladen Trust, RBGE Expedition Committee, British Lichen Society, William Steel Fund, and Oleg Polunin Memorial Fund to L. Olley.

\section{References}

Aptroot A, Feijen FJ. 2002 - Annotated checklist of the lichens and lichenicolous fungi of Bhutan. Fungal Diversity 21-48.

Asahina Y. 1955 - Lichens. In: Fauna and flora of Nepal Himalaya. Scientific results of the Japanese expeditions to Nepal Himalaya (1952-53). Fauna and Flora Research Society, Kyoto University, Kyoto, Japan. pp. 44-63.

Awasthi DD. 1960 - On a collection of macrolichens by the Indian expedition to Cho-Oyu, East Nepal. In: Proceedings of the Indian Academy of Science (Plant Sciences) , pp. 169-180.

Awasthi DD. 1965 - Catalogue of the lichens from India, Nepal, Pakistan and Ceylon. Beih Nova Hedw 17, 1-137.

Awasthi DD. 2007 - A compendium of the macrolichens from India, Nepal and Sri Lanka. Bishen Singh Mahendra Pal Singh, India.

Baniya CB, Solhøy T, Gauslaa Y, Palmer MW. 2010 - The elevation gradient of lichen species richness in Nepal. The Lichenologist 42, 83-96.

Cameron J. 1900 - The Gaelic names of plants. John MacKay, Glasgow.

Cornejo C, Chabanenko S, Scheidegger C. 2009 - Phylogenetic analysis indicates transitions from vegetative to sexual reproduction in the Lobaria retigera group (Lecanoromycetidae, Ascomycota). The Lichenologist 41, 275-284.

Cornejo C, Scheidegger C. 2015 - Multi-gene phylogeny of the genus Lobaria: Evidence of speciespair and allopatric cryptic speciation in East Asia. American Journal of Botany 102, 2058-2073.

Cornell HV, Lawton JH. 1992 - Species interactions, local and regional processes, and limits to the richness of ecological communities: a theoretical perspective. Journal of Animal Ecology 61, $1-12$.

Culberson CF. 1972 - Improved conditions for the identification of lichen products by a standardized thin-layer chromotagraphic method. Journal of Chromatography 72, 113-125.

Culberson CF, Kristinsson H. 1970 - A standardized method for the identification of lichen products. Journal of Chromatography 46, 85-93.

Dahlberg A, Mueller GM. 2011 - Applying IUCN red-listing criteria for assessing and reporting on the conservation status of fungal species. Fungal Ecology 4, 147-162.

Densmore F. 1939 - Nootka and Quileute music. Government Printing Office, Washington, USA.

Devkota S, Cornejo C, Werth S, Chaudhary RP. et al. 2014 - Characterization of microsatellite loci in the Himalayan lichen fungus Lobaria pindarensis (Lobariaceae). Applications in Plant Sciences 2.

DFRS 2015 - State of Nepal`s forest. Forest Resource Assessment (FRA) Nepal, Department of Forest Research and Survey (DFRS). Kathmandu, Nepal.

Din LB, Latiff A, Said IM, Elix JA. 2008 - Additional lichen records from Indonesia and Malaysia 
6. Lichens from Maliau Basin, Sabah, Malaysia Laily. Australian Lichenology 62, 3-5.

Edman M, Eriksson AM, Villard MA. 2008 - Effects of selection cutting on the abundance and fertility of indicator lichens Lobaria pulmonaria and Lobaria quercizans. Journal of Applied Ecology 45, 26-33.

Ellis CJ, Hope J. 2012 - Lichen epiphyte dynamics in Scottish Atlantic oakwoods - The effect of tree age and historical continuity. Scottish Natural Heritage Commissioned Report No. 426.

Galloway DJ. 1994 - Studies on the lichen genus Sticta (Schreber) Ach.: I. Southern South American species. The Lichenologist 26, 223-282.

Geiser LH, Dillman KL, Derr CC, Stensvold MC. 1994 - Lichens of Southeastern Alaska: An Inventory. USDA-Forest Service, Tonass National Forest / Stikine Area, Alaska 99833.

Guarrera PM, Lucchese F, Medori S. 2008 - Ethnophytotherapeutical research in the high Molise region Central-Southern Italy. Journal of Ethnobiology and Ethnomedicine 4, 1-11.

Hawksworth D, Kirk P, Sutton B, Pegler D. 1995 - Ainsworth and Bisby`s dictionary of the fungi. Cambridge University Press, Cambridge.

Hodgetts NG. 2000 - Interpreting the IUCN Red List categories and criteria for cryptogams. Forest Snow and Landscapre Research 75, 293-302.

Honegger R. 1991 - Functional aspects of the lichen symbiosis. Annual Review of Plant Physiology and Plant Molecular Biology 42, 553-578.

Hu S, Kong Y, But P. 1980 - An enumeration of the Chinese Materia Medica. The Chinese University Press, Hong Kong.

IUCN Standards and Petitions Subcommittee 2010 - Guidelines for Using the IUCN Red List Categories and Criteria. Version 8.1. Prepared by the Standards and Petitions Subcommittee in March 2010.

Jayalal U, Joshi S, Oh S-O, Kim JA et al. 2014 - The lichen genus Sticta in South Korea. Mycobiology 42, 6-11.

Jinong W, Huajie L. 2012 - Flora Lichenum Sinicorum. Vol 11. Peltigerales (1). Beijing: Science Press.

Johnson LM. 1997 - Health, wholeness, and the land: Gitksan traditional plant use and healing. PhD dissertation, University of Alberta, Canada.

Jordan WP. 1973 - The genus Lobaria in North America North of Mexico. Bryologist 76, 225-251.

Joshi M, Awasthi DD. 1982 - The lichen family Stictaceae in India and Nepal. Biological Memoirs 7, 165-190.

Joshi S, Upreti DK, Das P. 2016 - Lichen diversity assessment in Pindari Glacier Valley of Uttarakhand, India. Geophytology 41, 24-41.

Ju Y, Zhuo J, Liu B, C L. 2013 - Eating from the wild: diversity of wild edible plants used by Tibetans in Shangri-la region, Yunnan, China. Journal of Ethnobiology and Ethnomedicine, 9.

Katuwal HB, Basnet K, Khanal B, Devkota S et al. 2016 - Seasonal changes in bird species and feeding fuilds along elevational gradients of the Central Himalayas, Nepal. Plos One 11, 1-17.

Kim S. 1981 - Floral studies on the lichens in Korea. Bulletin of Kongju Teachers Coll 17, 279-305.

Kirk MP, Cannon PF, Minter DW, Stalpers JA. 2008 - Dictionary of the Fungi. CAB International, Wallingford.

Kurokawa S. 1967 - Foliose lichens collected by Dr. K. Yoda in the Rolwaling Himal, Nepal. Journal of College Arts and Sciences, Chiba University 5, 93-97.

de Lange PJ, Galloway DJ. 2015 - Lichen notes from the Kermadec Islands (ed. T. Trnski, H. A. Schlumpf). Bulletin of the Auckland Museum, 20, 141-170.

Li B, Lin Z-W, Sun H-D. 1991 - The chemical constitutents of four lichens from China. Acta Botanica Yunnanica 01, 81-84.

Lin C. 2013 - The lichen genus Lobaria at Shenmi Lake, Northeastern Taiwan. Collection and Research 26, 37-42.

Llano GA. 1948 - Economic uses of lichens. Economic Botany 2, 15-45.

Lücking R, Rivas Plata E, Chaves L, Umana L. et al. 2009 - How many tropical lichens are there... really? In: Bibliotheca Lichenologica (ed. A. Thell, M. R. D Seaward, T. Feuerer), J. Cramer 
in der Gebruder Borntraeger Verlagsbuchhandlung, Berlin - Stuttgart. pp. 399-418.

Lumbsch HT, Huhndorf S. 2010 - Myconet Volume 14, Part One. Outline of Ascomycota 2009. Fieldiana: Life and Earth Sciences, N.S. 1: 1-42.

McDonald T, Miadlikowska J, Lutzoni F. 2003 - The lichen genus Sticta in the Great Smoky Mountains: A phylogenetic study of morphological, chemical, and molecular data. The Bryologist 106, 61-79.

Miehe G. 1990 - Langtang Himal. Flora und Vegetation als Klimazeiger und-zeugen im Himalaya. Dissertationes Botanicae, Bd 158, J Cramer, Berlin, Stuttgart.

Millanes AM, Diederich P, Wedin M. 2016 - Cyphobasidium gen. nov., a new lichen-inhabiting lineage in the Cystobasidiomycetes (Pucciniomycotina, Basidiomycota, Fungi). Fungal Biology.

Molleman L, Boeve S, Wolf J, Oostermeijer G. et al. 2011 - Commercial harvesting and regeneration of epiphytic macrolichen communities in the Western Ghats, India. Environmental Conservation 38, 334-341.

Moncada B, Lücking R, Betancourt-Macuase L. 2013 - Phylogeny of the Lobariaceae (lichenized Ascomycota: Peltigerales), with a reappraisal of the genus Lobariella. The Lichenologist 45, 203-263.

Moncada B, Reidy B, Lücking R. 2014 - A phylogenetic revision of Hawaiian Pseudocyphellaria sensu lato (lichenized Ascomycota: Lobariaceae) reveals eight new species and a high degree of inferred. The Bryologist 117, 119-160.

Nascimbene J, Nimis PL, Ravera S. 2013 - Evaluating the conservation status of epiphytic lichens of Italy: A red list. Plant Biosystems - An International Journal Dealing with all Aspects of Plant Biology: Official Journal of the Societa Botanica Italiana 147, 898-904.

Neitlich PN, Mccune B. 1997 - Hotspots of epiphytic lichen diversity in two young managed forests. Conservation Biology 11, 172-182.

Oliver WRB. 1912 - List of lichens and fungi collected in the Kermadec Islands in 1908. Transactions of the New Zealand Institute 44, 86-87.

Olley L, Sharma LR. 2013 - A provisional checklist of the lichens of Nepal. Bulletin of Department of Plant Resources 35, 18-21.

Pandit G, Sharma B. 2012 - New records in the lichen family Lobariaceae from the Western Ghats of India. Mycosphere 3, 430-435.

Park YS. 1990 - The macrolichen flora of South Korea. The Bryologist 93, 105-160.

Pinokiyo A, Singh KP, Singh JS. 2008 - Diversity and distribution of lichens in relation to altitude within a protected biodiversity hot spot, north-east India. The Lichenologist 40, 47-62.

Radies D, Coxson D, Johnson C, Konwicki K. 2009 - Predicting canopy macrolichen diversity and abundance within old-growth inland temperate rainforests. Forest Ecology and Management 259, 86-97.

Rai SK, Sharma S, Shrestha KK, Gajurel JP et al. 2016 - Effects of the environment on species richness and composition of vascular plants in Manaslu Conservation Area and Sagarmatha region of Nepalese Himalaya. Banko Janakari 26, 3-16.

Ren MR, Wang XY, Koh YJ, Hur JS. 2012 - Taxonomic study of the lichen genus Lobaria in South Korea. Mycobiology 40, 1-7.

Scheidegger C, Goward T. 2002 - Monitoring lichens for conservation: Red lists and conservation action plans. In: Monitoring with Lichens - Monitoring Lichens (ed. P. L. Nimis, C. Scheidegger, P. A. Wolseley). Kluwer Academic Publishers. Printed in the Netherlands. pp. 163-181.

Scheidegger C, Nobis MP, Shrestha KK. 2010 - Biodiversity and livelihood in land-use gradients in an era of climate change - outline of a Nepal-Swiss research project. Botanica Orientalis: Journal of Plant Science 7, 7-17.

Scheidegger C, Stofer S, Dietrich M, Groner U. et al. 2000 - Estimating regional extinction probabilities and reduction in populations of rare epiphytic lichen-forming fungi. Forest, Snow and Landscape Research 75, 415-433.

Scheidegger C, Werth S. 2009 - Conservation strategies for lichens: insights from population 
biology. Fungal Biology Reviews 23, 55-66.

Sharma LR. 1979a - Contributions to the lichen flora of Nepal. PhD Dissertation, University of Lucknow, India.

Sharma LR. 1979b - Additions to the lichen flora of Nepal II. Geophytology 8, 247-248.

Sharma LR. 1995 - Enumeration of the lichens of Nepal. Biodiversity Profiles Project Technical Publications No. 3. Department of National Parks and Wildlife Conservation, Ministry of Forests and Soil Conservation. His Majesty`s Government of Nepal, Kathmandu.

Shrestha TB. 2008 - Classification of Nepalese forests and their distribution in protected areas. The Initiation 2, 1-9.

Shukla P, Bajpai R, Singh CP, Sharma N. et al. 2015 - Lichen diversity in alpine regions of eastern Sikkim with respect to long term monitoring programme of Indian Space Research Organization. Geophytology 1, 57-62.

Singh KP. 1981 - Macrolichens from Manipur. Biological Memoirs 6, 145-168.

Singh KP, Sinha GP. 2010- Indian Lichens : An annotated checklist. Botanical Survey of India, Ministry of Environment and Forests.

Sinha GP, Singh KP. 2005 - Macrolichens of Sikkim. Botanical Survey of India. Botanical Survey of India. Ministry of Environment and Forests, New Delhi, India.

Skaloud P, Friedl T, Hallmann C, Beck A. et al. 2016 - Taxonomic revision and species delimitation of coccoid green algae currently assigned to the genus Dictyochloropsis (Trebouxiophyceae, Chlorophyta). Journal of Phycology 52, 599-617.

Smith H. 1923 - Ethnobotany of the Menomini Indian. Bull Public Mus Milwaukee 4, 1-174.

Smith AL. 1931 - Lichens from Northern India. Trans Bot Mycol Soc 11, 189-196.

Søchting U. 1999 - Lichens of Bhutan -Biodiversity and Use. Botanical Institute, University of Copenhagen.

Søchting U. 2015 - Lichens. In: Nepal: An introduction to the natural history, ecology and human environment of the Himalayas (ed. G. Mihe, C. A. Pendry CA, R. P. Chaudhary). Edinburgh: Royal Botanic Garden Edinburgh. pp. 191-199.

Spribille T, Tuovinen V, Resl P, Vanderpool D. et al. 2016 - Basidiomycete yeasts in the cortex of ascomycete macrolichens. Science (New York, NY) 353 (6298), 488-92.

Turner NJ, Efrat BS. 1982 - Ethnobotany of the Hesquiat Indians of Vancouver Island.

Upreti D. 1998 - Diversity of lichens in India. In: Perspectives in environment (ed. S. Agrawal, J. Kaushik, K. Kaul, A. Jain). APH Publishing Corporation, New Delhi. pp. 71-79.

Upreti DK, Negi HR. 1996 - Folk use of Thamnolia vermicularis (Swartz) Ach. in Lata Village of Nanda Devi Biosphere Reserve. Ethnobotany 8, 83-86.

Upreti DK, Ranjan M. 1988 - A note on some macrolichens from Thimpu district, Bhutan. Journal of Recent Advances in Applied Sciences 3, 426-432.

Wang L, Qian Z. 2013 - '中国药用地衣图鉴 [Zhong guo yao yong di yi tu jian = Illustrated medicinal lichens of China].' (Yunnan ke ji chu ban she, China)

Wei J. 1991 - An enumeration of lichens in China. Beijing: International Academic Publishers.

Wirtz N, Lumbsch HT, Green TGA, Türk R. et al. 2003 - Lichen fungi have low cyanobiont selectivity in maritime Antarctica. New Phytologist 160, 177-183.

Yoshimura I. 1969 - Lichenological Notes 2-6. Journal of the Hattori Botanical Laboratory 32, 6778.

Yoshimura I. 1971 - The genus Lobaria of Eastern Asia. Journal of the Hattori Botanical Laboratory 34, 231-364. 